\title{
Evolution of soft palate surgery techniques for obstructive sleep apnea patients: A comparative study for single-level palatal surgeries
}

\author{
Mohamed S. Rashwan ${ }^{1}$ (D) | Filippo Montevecchi ${ }^{2}$ | Giovanni Cammaroto ${ }^{2,3}$ | \\ Mohamed Badr el Deen $^{1}$ | Nagi Iskander ${ }^{1}$ | Diaa El Hennawi ${ }^{1}$ | \\ Mohammed El Tabbakh $^{1}$ (D) | Giuseppe Meccariello ${ }^{2}$ | Riccardo Gobbi ${ }^{2}$ | \\ Francesco Stomeo ${ }^{4}$ | Claudio Vicini ${ }^{2,4}$
}

${ }^{1}$ Faculty of Medicine, Department of Otorhinolaryngology, Suez Canal University Teaching Hospitals, Ismailia, Egypt

${ }^{2}$ Head and Neck Department - ENT \& Oral Surgery Unit, G.B. Morgagni - L. Pierantoni Hospital, Forlì - ASL of Romagna, Italy

${ }^{3}$ Department of Otorhinolaryngology, University of Messina, Messina, Italy

${ }^{4}$ ENT \& Audiology Department, University of Ferrara, Ferrara, Italy

Correspondence

Mohamed S. Rashwan, Department of Otolaryngology, Faculty of Medicine, Suez Canal University Teaching Hospitals, Ismailia, Egypt.

Email: m_salaheldin@med.suez.edu.eg
Objectives: To compare the results of tissue preservation techniques of soft palate surgeries including expansion sphincter pharyngoplasty (ESP) and barbed reposition pharyngoplasty (BRP) for patients suffering from obstructive sleep apnoea (OSA) with the traditional uvulopalatopharyngoplasty (UPPP).

Design: Interventional comparative study.

Setting: Morgagni-Pierantoni Hospital.

Participants: Seventy-five patients were included in the study, divided into three groups with 25 patients per group: UPPP, ESP or BRP.

Main outcomes measures: Polysomnography was carried out for all patients preand postoperatively; the postoperative results were recorded at least 6 months after surgery. All patients were assessed preoperatively using drug-induced sleep endoscopy. Epworth Sleepiness Scale and body mass index (BMI) were registered for all patients before and after surgery.

Results: The mean of pre- and postoperative differences of apnoea-hypopnoea index values was higher in BRP group than ESP: $15.76 \pm 14.5$ Vs $10.13 \pm 5.3$; $P<.05$ and UPPP groups: $15.76 \pm 14.5$ vs $6.08 \pm 5.5 ; P<.0005$. The mean of differences of oxygen desaturation index values was higher in BRP group than UPPP group: $15.09 \pm 17.6$ vs $7.13 \pm 6.8 ; P<.0005$, but not significantly higher than ESP group: $15.09 \pm 17.6$ vs $6.48 \pm 7.9 ; P>.05$. The mean of differences of ESS values was higher in BRP group than ESP group: $5.52 \pm 4.1$ vs $4.84 \pm 3.3 ; P<.005$ and UPPP groups: $5.52 \pm 4.1$ vs $1.36 \pm 1.9 ; P<.005$. Finally, the pre- and postoperative means of differences of lowest oxygen saturation values were not statistically significant among the three groups $(P>.05)$.

Conclusion: Barbed reposition pharyngoplasty (BRP) can be considered an effective procedure on the basis of the postoperative outcomes. ESP still proves to be a good technique especially when performed by experienced surgeons. Both techniques proved to be superior to UPPP. 


\section{1 | INTRODUCTION}

Obstructive sleep apnoea (OSA) is a common health problem affecting a large number of people all over the world with great psychological and physiological burdens. The overall population prevalence ranged from $9 \%$ to $38 \%$ at apnoea-hypopnoea index $(\mathrm{AHI}) \geq 5$, being higher in men. It increases with age and, in some elderly groups, was as high as $90 \%$ in men and $78 \%$ in women. The prevalence decreases as the severity increases to range from $6 \%$ to $17 \%$ at $\mathrm{AHI}$ $\geq 15$, being as high as $49 \%$ in the advanced ages. OSA prevalence is directly proportional to the body mass index (BMI), so the disease is more common among obese people. ${ }^{1}$

Several degrees and patterns of collapse of the upper airway (UA) during sleep have been described in patients with OSA. In our study, attention is mainly focused on soft palatal collapse which is considered the most common site causing OSA and snoring. ${ }^{2}$

Uvulopalatopharyngoplasty (UPPP) is the most commonly performed surgical procedure for treatment of OSA. The challenge of this procedure is to determine the limits of soft tissue resection to be effective. Moreover, the preservation of the levator palatine muscle is mandatory to maintain normal speech and avoid nasal regurgitation. $^{3}$

Lateral pharyngeal muscle wall collapse has been demonstrated to be pivotal in the pathogenesis of OSA in imaging studies. ${ }^{4}$ Druginduced sleep endoscopy (DISE) aided more in the understanding of the effect of the circumferential collapse at the velum level in failure of palatal surgery. ${ }^{5}$

The lateral pharyngoplasty (LP), firstly described by Cahali, ${ }^{6}$ was aimed at addressing the lateral pharyngeal wall collapse in patients with OSA but it carried severe postoperative dysphagia as relevant drawback. LP showed better PSG results despite not having differences on the postoperative volume of the UA, underlying the importance of changing the mechanical properties rather than removing tissue.

Another frequently used technique is expansion sphincter pharyngoplasty (ESP), which involves rotation of the palatopharyngeus muscle and its anchorage to the pterygoid hamulus, a partial uvulectomy and closure of the anterior and posterior tonsillar pillars. $^{7}$

A new palatal surgical technique that has been recently described in the literature is the barbed reposition pharyngoplasty (BRP). ${ }^{8}$ This procedure allows surgeons to achieve widening and stiffening of the nasopharyngeal inlet without any tissue sacrifice by means of a bidirectional barbed suture that is inserted through the fibro-muscular tissues of the soft palate and the posterior tonsillar pillars, and tightened around three steady holds: the posterior nasal spine and the two pterygoid hamulii lateral to the pterygomandibular raphe. 8,9

Based on the above facts, the rational of this study stands upon the comparison between non-resective techniques of soft palate surgeries (ESP and BRP) with UPPP to prove which technique allows the best functional outcomes in OSA patients with single-level collapse.

\section{Keypoints}

- Barbed Reposition Pharyngoplasty

- Expansion Sphincter Pharyngoplasty

- Uvulopalatopharyngoplasty

- Obstructive sleep apnea

- Lateral pharyngeal wall collapse

\section{2 | METHODS}

Local ethics committees or institutional review boards approved the study. The medical charts of patients of the three groups of patients at the department of Otolaryngology and Head-Neck Surgery, Morgagni-Pierantoni Hospital, Forlì, Ausl della Romagna, Italy, between November 2004 and March 2016 were evaluated retrospectively. Seventy-five patients were included in the study and divided into three groups with twenty-five patients per group: UPPP, ESP or BRP. Patients were recruited from the medical records randomly according to the availability of the pre and postoperative data in addition to those performing only palate and nasal surgery; we tried to match the three groups as much as possible in terms of the $\mathrm{AHI}$, BMI and DISE findings. We considered the BRP group as Gold Standard and performed multiple comparisons using the BRP group as control group versus ESP and UPPP groups.

\subsection{Full medical history}

Full medical history with preoperative and postoperative Italian version of Epworth Sleepiness Scale (ESS) was collected to all patients. ${ }^{10}$

\section{2 | Physical examination}

Physical examination was performed according to OSA phenotypes which include anatomical and functional phenotypes.

Anatomical phenotypes: Modified Mallampati index, ${ }^{11}$ Friedman Staging System ${ }^{12}$ and Tucker Woodson description for palatal position. ${ }^{13}$

Functional phenotypes: Müller manoeuver ${ }^{14}$ and DISE, ${ }^{15}$ preoperative evaluation with DISE was performed for all patients to confirm the single-level palatal obstruction. DISE was performed by means of a flexible rhinopharyngolaryngoscope in the operating theatre using target-controlled infusion ( $\mathrm{TCl}$ ) of propofol to achieve a complete evaluation of the UA collapse and especially of the lateral pharyngeal walls. Patients were classified according to the NOHL classification system according to Vicini et al. ${ }^{16}$ Preoperative and 6 months postoperative polysomnographies evaluating $\mathrm{AHI}$, oxygen desaturation index (ODI) and lowest oxygen saturation (LOS) were performed to compare the results within each group and between the three groups. All the sleep studies were carried out in an unattended way by means of a 
Polymesam Unattended Device 8-channel; reviewed and scored by the same expert in sleep medicine according to the American Academy of Sleep Medicine Guidelines 2007. ${ }^{17}$ Body mass index (BMI) was calculated for all patients before and after surgery. ${ }^{18}$

\section{3 | Inclusion criteria}

Patients suffering from OSA planned for palate surgery and tonsillectomy, with nasal surgery (Septoturbinoplasty), aged between 21 and 65 years old and $\mathrm{BMI} \leq 35$.

\subsection{Exclusion criteria}

Patients treated with multilevel surgery including tongue base reduction or epiglottoplasty, unfit for general anaesthesia, allergy to propofol, patients with significant craniofacial anomalies, patient refusal, patients who underwent previous OSA surgeries and pregnant women.

\section{5 | Statistical analysis}

Data analyses were performed in $\mathrm{R}$ using Bioconductor libraries and R statistical packages (http://www.r-project.org/, R Development Core Team, 2008). Wilcoxon signed-rank test was used to compare pre- and postoperative outcomes within each group (homogeneity between groups was tested), while ANOVA test was used to compare the outcomes between the groups. Pre- and postoperative mean differences of AHI, ODI, ESS and LOS (delta = preoperative - postoperative) were compared between groups.

\section{3 | RESULTS}

Preoperative values in all groups are presented in Table 1. Pre- and postoperative mean differences of $\mathrm{AHI}, \mathrm{ODI}$ and ESS values were calculated, and statistically significant reduction in these parameters was seen in the three groups $(P<.05)$, Table 2 . On the other hand, LOS decreased significantly in BRP and ESP groups, but not in UPPP group, Figure 1.

The results of the three groups analysis showed that $\mathrm{AHI}$ values decreased more significantly in BRP group than ESP $(15.76 \pm 14.5$ vs $10.13 \pm 5.3 ; \quad P<.05)$ and UPPP groups $(15.76 \pm 14.5$ vs $6.08 \pm 5.5 ; P<.0005)$. The mean of differences of ODI values was higher in BRP group than UPPP group $(15.09 \pm 17.6$ vs $7.13 \pm 6.8$; $P<.0005)$ but not than ESP group $(15.09 \pm 17.6$ vs $6.48 \pm 7.9$; $P>$.05). Furthermore, ESS values decreased more significantly in
TABLE $2 P$ values of within groups analysis (Wilcoxon test)

\begin{tabular}{llllc} 
Groups & Delta - AHI & Delta - ODI & Delta - ESS & Delta - LOS \\
BRP & $<.0001$ & .0001 & $<.0001$ & .0018 \\
ESP & $<.0001$ & .0005 & $<.0001$ & $<.0001$ \\
UPPP & $<.0001$ & $<.0001$ & .0001 & .1084 \\
\hline
\end{tabular}

BRP group than ESP $(5.52 \pm 4.1$ vs $4.84 \pm 3.3 ; P<.005)$ and UPPP groups $(5.52 \pm 4.1$ vs $1.36 \pm 1.9 ; P<.005)$. Finally, the pre- and postoperative means of differences of LOS values were not statistically significant among the three groups $(P>.05)$, Figure 2 . No statistically significant difference in the pre- and postoperative BMI data between the three groups was observed.

\section{4 | DISCUSSION}

Different palatal techniques have been introduced along the last two decades, especially after the evolution of palatal surgeries focused on the lateral pharyngeal wall collapse, from the first lateral pharyngoplasty $^{6}$ to the newest BRP. ${ }^{9}$ Although a lot of publications describing the drawbacks of UPPP can be found in scientific literature, there are still many articles highlighting the improvement of $\mathrm{AHI}$ after UPPP, not taking postoperative complications into consideration and not comparing its results with the outcomes of other palatal procedures.

Our study was performed on patients undergoing palate surgery with tonsillectomy combined with nasal surgery. The idea was to evaluate the results of the three chosen techniques without the association to surgical procedure addressed to other anatomical districts.

Our team chose to compare both ESP and BRP as they are the two most used techniques in our department, with the most performed palatal procedure all over the world (UPPP).

From our results, it appears that both BRP and ESP may allow to achieve better results than UPPP in terms of PSG parameters and ESS score.

The reported success of UPPP as an OSA treatment was between $16 \%$ and $83 \%$ depending on the definition of a positive outcome and selection of patients. ${ }^{19}$ Some authors have defined surgical success after UPPP as a $50 \%$ reduction in the $\mathrm{AHI}$, whereas others combine this criterion with an absolute $\mathrm{AHI}$ of 20 or less. ${ }^{20}$, 21 The success rate of UPPP in unselected patients was around $40 \%,{ }^{22}$ and this is the main reason why it is not recommended as a standard treatment. ${ }^{23}$ Side effects include difficulty swallowing/nasal regurgitation, taste disturbances and voice changes. ${ }^{24}$

TABLE 1 Preoperative values (means \pm standard deviation). No differences between groups were found

\begin{tabular}{lllll} 
Group & AHI & ODI & ESS & LOS \\
BRP & $25.58 \pm 14.60$ & $24.39 \pm 17.73$ & $9.28 \pm 3.10$ & $80.56 \pm 7.51$ \\
ESP & $19.14 \pm 9.66$ & $16.30 \pm 8.95$ & $8.96 \pm 3.36$ & $86.52 \pm 4.64$ \\
UPPP & $18.96 \pm 17.79$ & $17.56 \pm 16.64$ & $8.80 \pm 3.23$ & $77.60 \pm 12.04$ \\
\hline
\end{tabular}



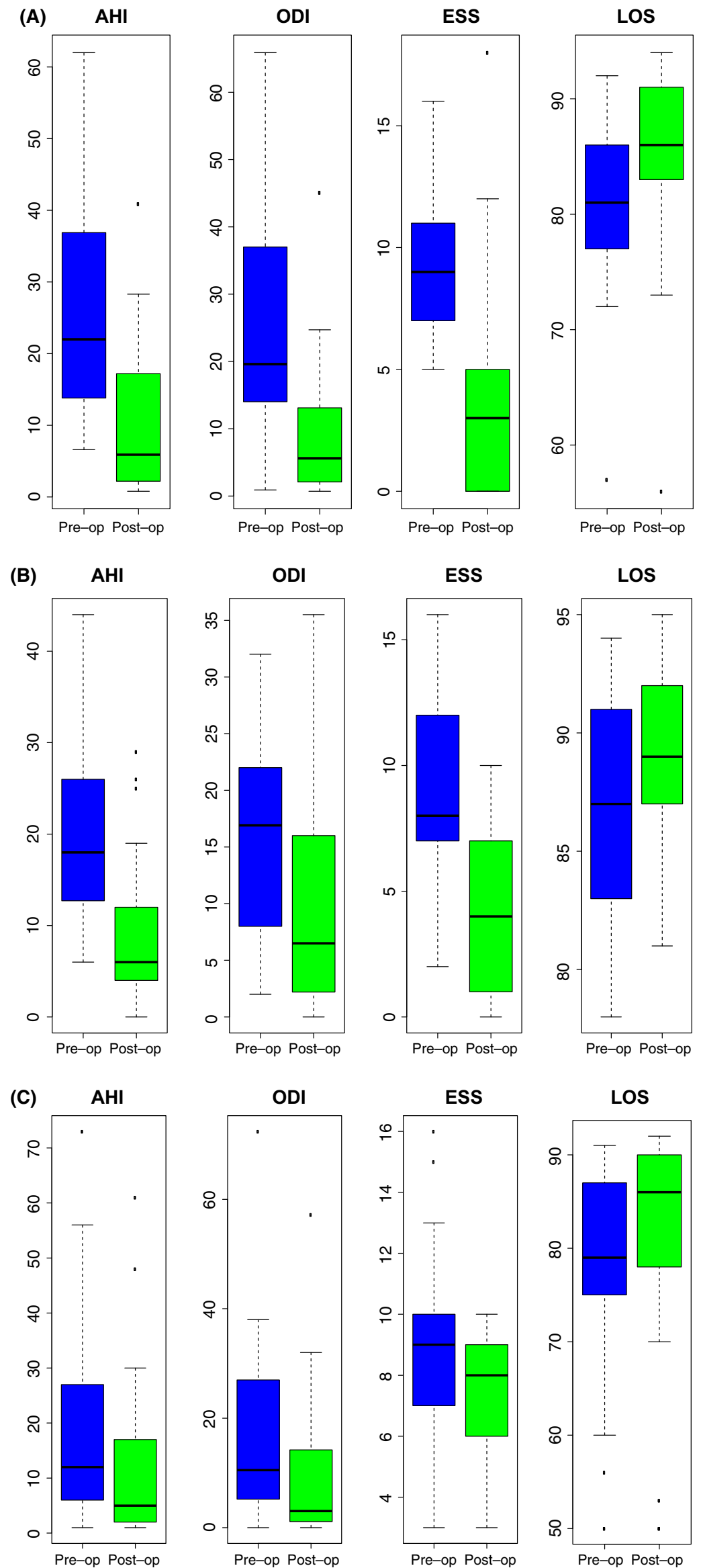

FIGURE 1 A, Boxplots represent AHI, ODI, ESS and LOS values of post-surgery time and pre-surgery time in BRP group. B, Boxplots represent AHI, ODI, ESS and LOS values of post-surgery time and presurgery time in ESP group. C, Boxplots represent $A H I, O D I, E S S$ and LOS values of post-surgery time and pre-surgery time in UPPP group. The bottom and top of the box are the first and the third quartiles, and the band inside the box is the median; whiskers represent $1^{\circ}$ and $99^{\circ}$ percentiles; values that are lower and greater are shown as circles 
(A)

OVERALL AHI

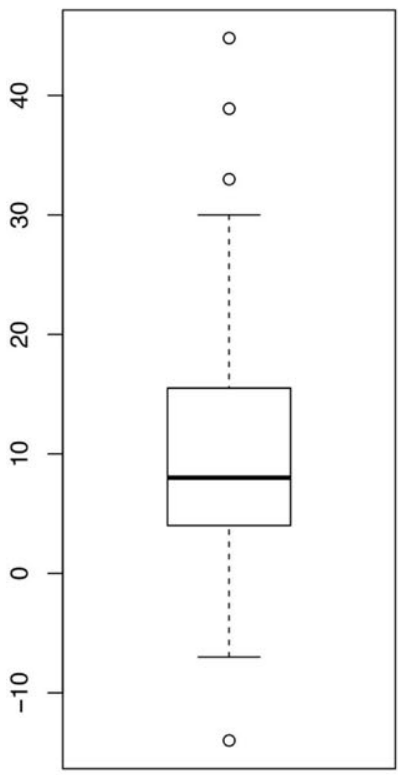

(A) OVERALL ODI

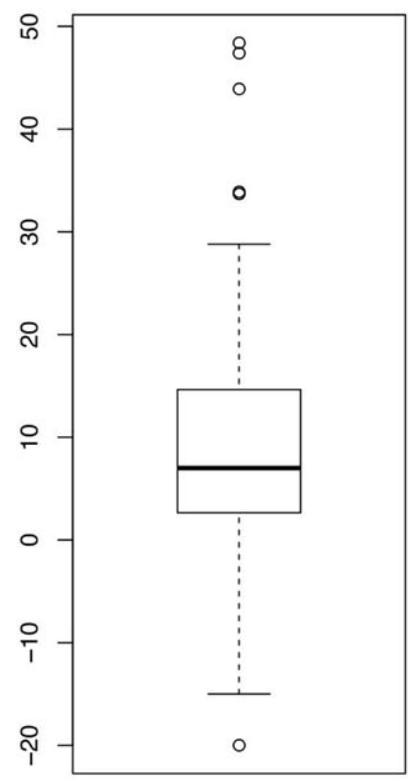

(B)

AHI

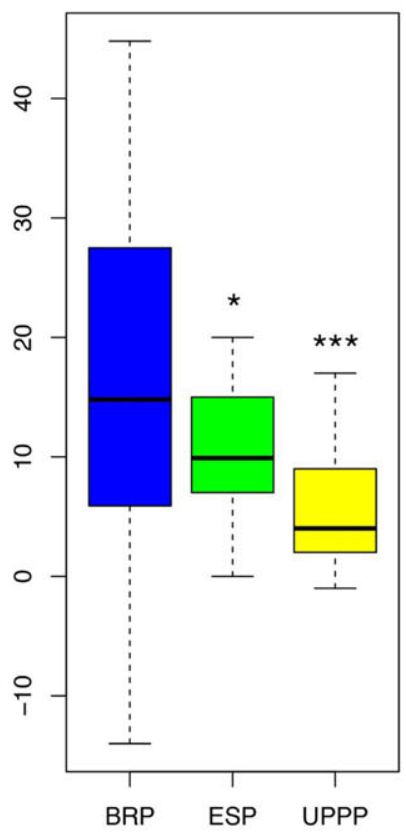

(B)

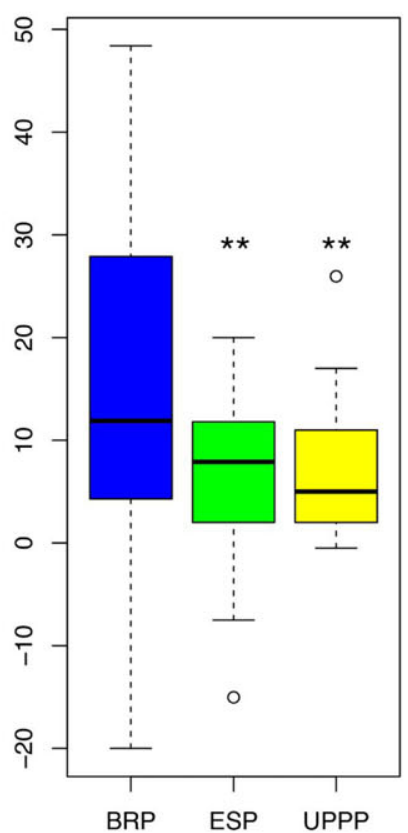

(A) OVERALL ESS

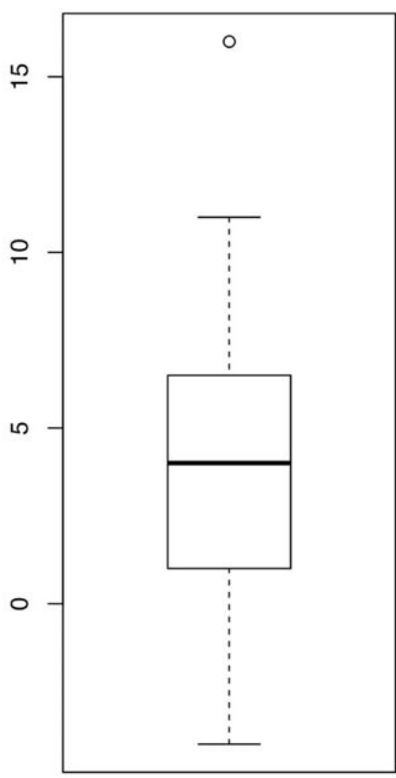

(B) ESS
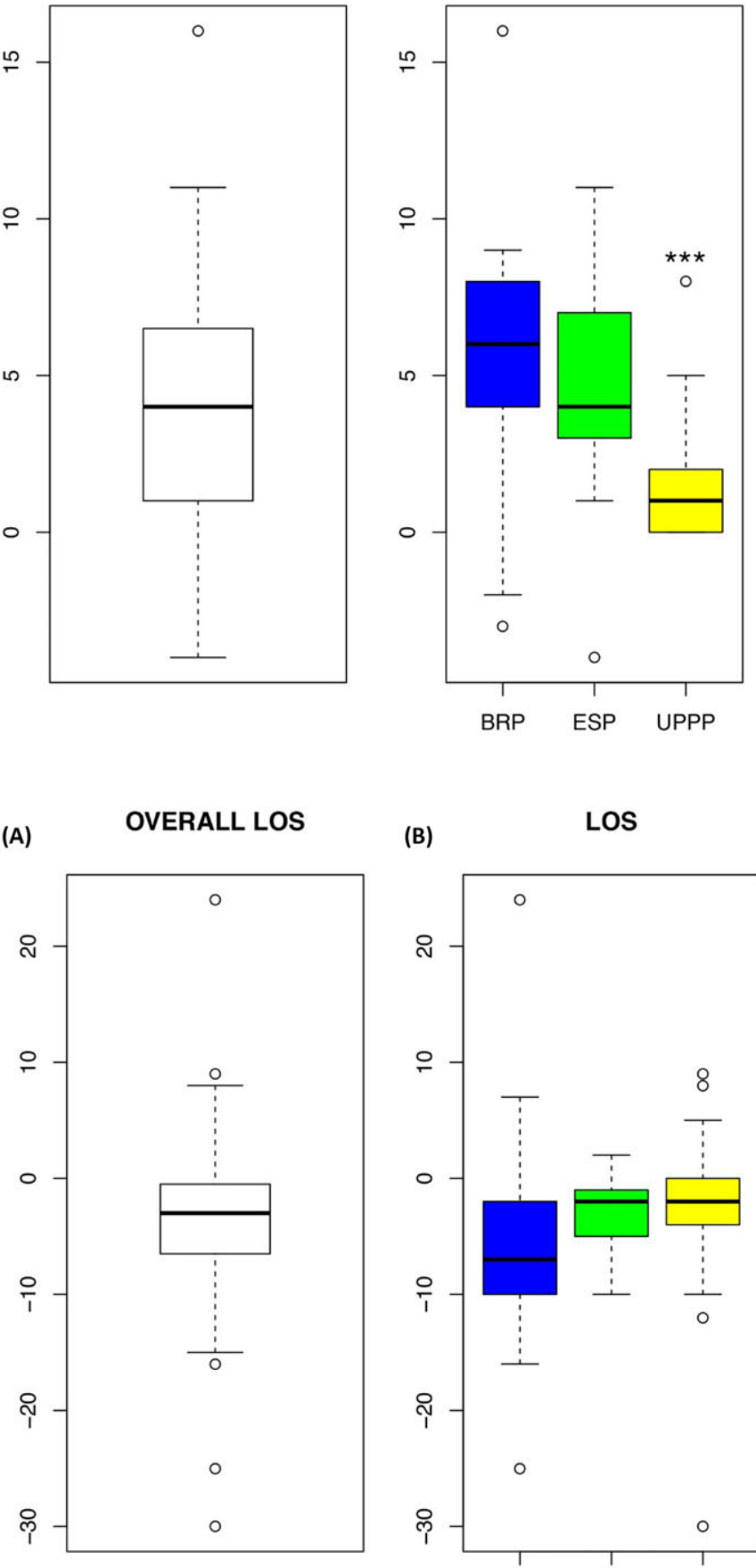

(B)

LOS

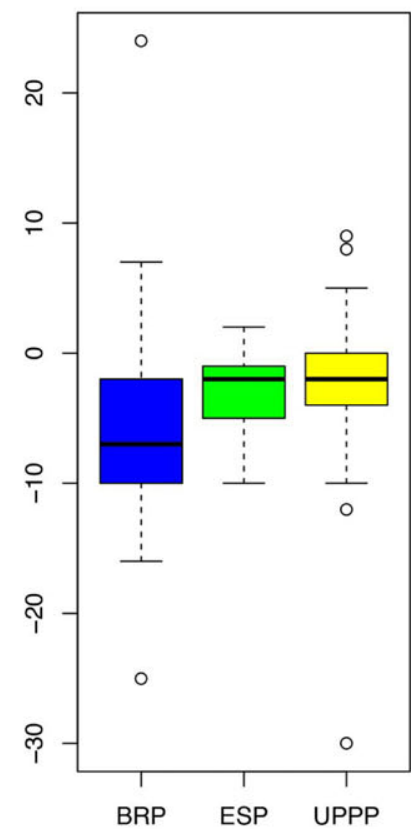

FIGURE 2 A, Overall mean of differences of AHI, ODI, ESS and LOS values between post-surgery time and pre-surgery time. B, Difference of AHI, ODI, ESS and LOS values between post-surgery time and pre-surgery time among three groups as visualised by the boxplot. The bottom and top of the box are the first and the third quartiles, and the band inside the box is the median; whiskers represent $1^{\circ}$ and $99^{\circ}$ percentiles; values that are lower and greater are shown as circles, asterisks represent significance $(\mathrm{P}$-value $<.05)$

In 2007, Pang and Woodson concluded that their ESP is a safe and effective procedure in patients with lateral pharyngeal wall collapse, superior to UPPP, and with less short- and long-term complications. $^{7}$ In 2016, Pang et al. as well could prove through a systematic review and metanalysis that ESP provides better outcomes than other traditional methods of palatal surgeries. ${ }^{25}$ In line with these results, Vicini et al. concluded that as a part of multilevel procedure, including conventional nasal surgery and robotic surgery, ESP seems to be superior to UPPP. ${ }^{26}$ Our results were consistent with these studies as the improvement in the postoperative 
$\mathrm{AHI}, \mathrm{ODI}$ and ESS was higher in the BRP group followed by the ESP group and the UPPP group.

In 2017, Cammaroto et al. showed similar results in patients treated with palatal surgery combined with transoral robotic surgery (TORS). The study showed no major difference between the BRP and the ESP groups, although both techniques proved to be more effective than UPPP in a multilevel setting.

However, BRP was seen to be a quicker and easier technique and provided minimal blood loss and better preservation of the mucosal and muscular tissues in comparison with ESP and, of course UPPP. ${ }^{9,27}$

We owe the credit of the better results of both BRP and ESP over UPPP to lateral widening in the retropalatal space provided by the upward and lateral rotation of the palatopharyngeus muscle. Moreover, BRP allows a more anterior soft palate displacement due to the lateral anchoring of the sutures on the pterygomandibular raphe.

Finally, the concentric scar that usually occurs in UPPP is better avoided to avoid one of the worst complications, that is velopharyngeal stenosis, as mentioned in several case reports. ${ }^{28}$

\subsection{Limitations of the study}

More patients should be included in future studies, and prospective randomised control study design would provide us more reliable data. Longer follow-up for the patients will enable us to highlight the short- and long-term results for better judgment on the surgical outcome.

\section{5 | CONCLUSIONS}

In conclusion, BRP and ESP seem to be more effective than UPPP in a single-level surgical setting. However, being quick, easy to learn and less invasive, BRP is a safe, effective and promising option for treatment of patients with OSA.

\section{CONFLICT OF INTEREST}

None to declare.

\section{ORCID}

Mohamed S. Rashwan (iD http://orcid.org/0000-0001-8233-196X

Mohammed El Tabbakh iD http://orcid.org/0000-0003-4207-9725

\section{REFERENCES}

1. Senaratna C. V., et al. Prevalence of obstructive sleep apnea in the general population: A systematic review. Sleep Med Rev. 2016;34:7081.

2. Eichler $\mathrm{C}$, et al. Does drug-induced sleep endoscopy change the treatment concept of patients with snoring and obstructive sleep apnea? Sleep Breath. 2013;17:63-68.
3. Dickson RI, Blokmanis A. Treatment of obstructive sleep apnea by uvulopalatopharyngoplasty. Laryngoscope. 1987;97:1054-1059.

4. Schwab RJ, et al. Dynamic upper airway imaging during awake respiration in normal subjects and patients with sleep disordered breathing. Am Rev Respir Dis. 1993;148:1385-1400.

5. Koutsourelakis I, et al. Surgery for obstructive sleep apnea: sleep endoscopy determinants of outcome. Laryngoscope. 2012;122:25872591.

6. Cahali MB. Lateral pharyngoplasty: a new treatment for obstructive sleep apnea hypopnea syndrome. Laryngoscope. 2003;113:19611968.

7. Pang KP, Woodson BT. Expansion sphincter pharyngoplasty: a new technique for the treatment of obstructive sleep apnea. Otolaryngol Head Neck Surg. 2007;137:110-114.

8. Mantovani M, et al. The velo-uvulo-pharyngeal lift or "roman blinds" technique for treatment of snoring: a preliminary report. Acta Otorhinolaryngol Ital. 2012;32:48-53.

9. Vicini $\mathrm{C}$, et al. Barbed reposition pharyngoplasty (BRP) for OSAHS: a feasibility, safety, efficacy and teachability pilot study. "We are on the giant's shoulders". Eur Arch Otorhinolaryngol. 2015;272:3065-3070.

10. Vignatelli L, et al. Italian version of the Epworth sleepiness scale: external validity. Neurol Sci. 2003;23:295-300.

11. Nuckton TJ, et al. Physical examination: Mallampati score as an independent predictor of obstructive sleep apnea. Sleep. 2006;29:903-908.

12. Friedman $\mathrm{M}$, et al. Clinical staging for sleep-disordered breathing. Otolaryngol Head Neck Surg. 2002;127:13-21.

13. Woodson BT. A method to describe the pharyngeal airway. Laryngoscope. 2015;125:1233-1238.

14. Terris DJ, et al. Reliability of the Muller maneuver and its association with sleep-disordered breathing. Laryngoscope. 2000;110:1819-1823.

15. De Vito A, Carrasco Llatas M, Vanni A, et al. European position paper on drug-induced sedation endoscopy (DISE). Sleep Breath. 2014;18:453-465.

16. Vicini $\mathrm{C}$, et al. The nose oropharynx hypopharynx and larynx (NOHL) classification: a new system of diagnostic standardized examination for OSAHS patients. Eur Arch Otorhinolaryngol. 2012;269:12971300.

17. Morgenthaler $\mathrm{T}$, et al. Practice parameters for the use of actigraphy in the assessment of sleep and sleep disorders: an update for 2007. Sleep. 2007;30:519-529.

18. Hoff PT, et al. Body mass index predicts success in patients undergoing transoral robotic surgery for obstructive sleep apnea. ORL $J$ Otorhinolaryngol Relat Spec. 2014;76:266-272.

19. Yousuf $A$, et al. Clinical predictors for successful uvulopalatopharyngoplasty in the management of obstructive sleep apnea. Int J Otolaryngol. 2013;2013:290265.

20. Dattilo DJ, Drooger SA. Outcome assessment of patients undergoing maxillofacial procedures for the treatment of sleep apnea: comparison of subjective and objective results. J Oral Maxillofac Surg. 2004;62:164-168.

21. Megwalu UC, Piccirillo JF. Methodological and statistical problems in uvulopalatopharyngoplasty research: a follow-up study. Arch Otolaryngol Head Neck Surg. 2008;134:805-809.

22. Sher $A E$, et al. The efficacy of surgical modifications of the upper airway in adults with obstructive sleep apnea syndrome. Sleep. 1996;19:156-177.

23. Sundaram S., et al. Surgery for obstructive sleep apnoea. Cochrane Database Syst Rev. 2005; 4: CD001004

24. Franklin KA, et al. Effects and side-effects of surgery for snoring and obstructive sleep apnea-a systematic review. Sleep. 2009;32: 27-36.

25. Pang KP, et al. Expansion sphincter pharyngoplasty for the treatment of OSA: a systemic review and meta-analysis. Eur Arch Otorhinolaryngol. 2016;273:2329-2333. 
26. Vicini $\mathrm{C}$, et al. Combined transoral robotic tongue base surgery and palate surgery in obstructive sleep apnea-hypopnea syndrome: expansion sphincter pharyngoplasty versus uvulopalatopharyngoplasty. Head Neck. 2014;36:77-83.

27. Cammaroto $\mathrm{G}$, et al. Palatal surgery in a transoral robotic setting (TORS): preliminary results of a retrospective comparison between uvulopalatopharyngoplasty (UPPP), expansion sphincter pharyngoplasty (ESP) and barbed repositioning pharyngoplasty (BRP). Acta Otorhinolaryngol Italy. 2017; [Epub ahead of print].

28. Katsantonis GP, et al. Nasopharyngeal complications following uvulopalatopharyngoplasty. Laryngoscope. 1987;97:309-314.
How to cite this article: Rashwan MS, Montevecchi F, Cammaroto G, et al. Evolution of soft palate surgery techniques for obstructive sleep apnea patients: $A$ comparative study for single-level palatal surgeries. Clin Otolaryngol. 2017;00:1-7. https://doi.org/10.1111/coa.13027 